\title{
Spread Through Air Spaces (STAS) in Lung Cancer: A Multiple-Perspective and Update Review
}

This article was published in the following Dove Press journal: Cancer Management and Research

\section{Meng Jia \\ Shili Yu \\ Hongwen Gao \\ Ping-Li Sun (D)}

Department of Pathology, The Second Hospital of Jilin University, Changchun, Jilin, People's Republic of China
Correspondence: Ping-Li Sun

Tel +86 43I-8II36833

Email pinglisun@naver.com
Background: Spread through air spaces (STAS) is a spreading phenomenon of lung cancers, which is defined as tumor cells within air spaces in the lung parenchyma beyond the edge of the main tumor. To date, several articles have reviewed the studies concerning the significance of STAS; however, most articles focused on the prognosis without summarizing the significance of STAS on other aspects. In this review, we comprehensively summarized the current literature related to STAS, so as to explore the clinical significance of STAS from multiple perspectives.

Main Body: This section provided a comprehensive overview of the significance of STAS from multiple perspectives and summarized current controversies and challenges in the diagnosis and clinical application.

Conclusion: STAS is a conspicuous spreading phenomenon of lung cancers indicating worse prognosis; nevertheless, the treatment strategy for patients with STAS remains to be discussed. Further studies are needed to elaborate whether a STAS-positive patient who underwent limited resection needs a second operation or postoperative adjuvant treatment. Meanwhile, the internal mechanism of STAS formation is largely undiscovered. Whether the capability of detachment-migration-reattachment in STAS tumor cells is achieved at the time of primary tumorigenesis or in the progress of tumor development needs to be studied, and the related signal pathways or genetic alterations need to be explored. With this information, it may be possible to improve the prognosis of patients with STAS-positive lung cancers.

Keywords: spread through air spaces, non-small cell lung cancer, adenocarcinoma, squamous cell carcinoma

\section{Introduction}

Spread through air spaces (STAS) is a spreading phenomenon of lung cancers, which was firstly named by Kadota and colleagues in $2015 .^{1}$ STAS is defined as tumor cells within air spaces in the lung parenchyma beyond the edge of the main tumor. STAS was initially observed in adenocarcinomas (ADC) (Figure 1A and B); however, with recent in-depth studies, STAS was identified in other types of lung cancer including squamous cell carcinoma (SQCC) (Figure $1 \mathrm{C}$ and D), ${ }^{2-4}$ pleomorphic carcinoma, ${ }^{5}$ invasive mucinous adenocarcinoma (IMA) (Figure 1E and F), ${ }^{6,7}$ neuroendocrine tumors ${ }^{8,9}$ and lymphoepithelioma-like carcinoma. ${ }^{10}$ To date, several articles have reviewed the studies concerning the significance of STAS; ${ }^{11-13}$ however, most of these articles focused on the correlation between STAS and clinicopathologic features or prognosis, and few articles summarized the 

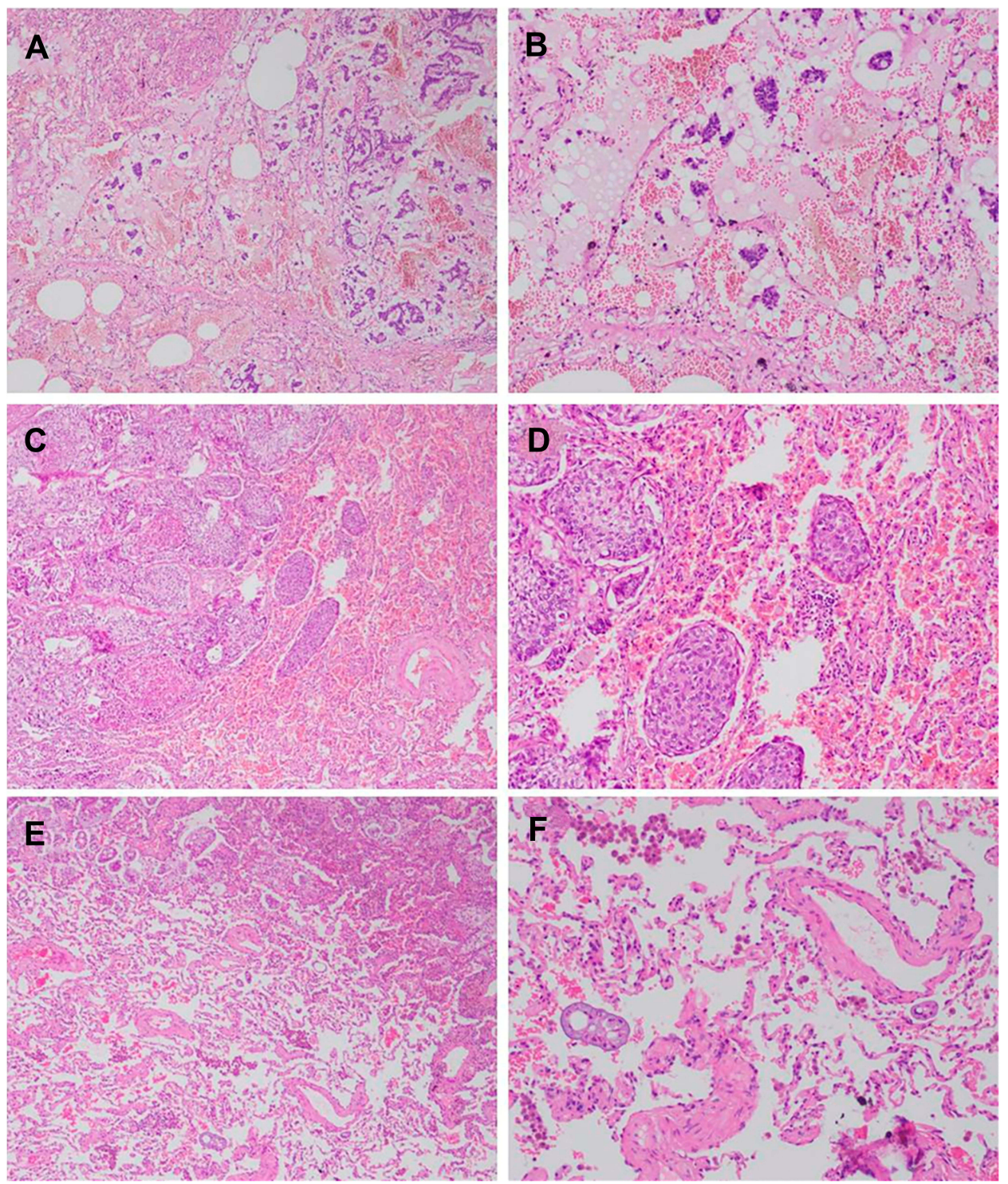

Figure I The representative pictures of STAS. (A-B) STAS in ADC; (C-D) STAS in SQCC; (E-F) STAS in IMA (Hematoxylin-eosin staining, (A, C, E) ×40, (B, D, F) × 100).

significance of STAS on other aspects. Meanwhile, since some new studies have been published, few articles contained the latest progress about this topic. In this review, we comprehensively summarized the current literature related to STAS, so as to explore the clinical significance of STAS from multiple perspectives.

\section{Historical Synonyms of STAS}

The phenomenon of free lung tumor cells spreading in the lung parenchyma has long been described before formally named by Kadota and colleagues in 2015. ${ }^{1}$ In 2000, Giraud et al used the term "aerogenous dissemination" to describe the presence of free tumor cells in the alveolar lumen. ${ }^{14}$ In 2013, Onozato and colleagues described large collections of tumor cells isolated within alveolar spaces and named these tumor cells "tumor islands". ${ }^{15}$ These descriptions shared similar meaning with the term "STAS". In 2015, Kadota and colleagues firstly named this phenomenon "STAS", and this term has been widely used since then.

\section{Clinicopathologic Features and Prognosis of STAS}

Since 2015, a variety of studies have focused on the association between STAS and clinicopathologic characteristics as well as prognosis, and the results are summarized in Table 1 briefly. In ADC (all stages), the incidence of STAS was from $28.2 \%{ }^{16}$ to $51.4 \% .{ }^{17,18}$ 
Table I Significance of STAS in Different Histological Types of Lung Cancer

\begin{tabular}{|c|c|c|c|c|}
\hline $\begin{array}{l}\text { Histological } \\
\text { Types }\end{array}$ & Positive Rate & Clinical Parameters & Pathologic Parameters & Prognosis \\
\hline ADC & $28.2 \%$ to $51.4 \%$ & $\begin{array}{l}\text { Old age (>65 years), male } \\
\text { sex, smoking, abnormal } \\
\text { serum carcinoembryonic } \\
\text { antigen level }\end{array}$ & $\begin{array}{l}\text { Larger tumor size, non-lepidic (micropapillary/ } \\
\text { solid) predominant, cribriform component, } \\
\text { moderate/poor differentiation, visceral pleural } \\
\text { invasion, lymphovascular invasion, presence of } \\
\text { nodal and distant metastasis, higher T, N and } \\
\text { pathological stage }\end{array}$ & Worse RFS and OS \\
\hline SQCC & $19.1 \%$ to $40.3 \%$ & NA & $\begin{array}{l}\text { Larger tumor size, lymphovascular invasion, } \\
\text { tumor necrosis, high-grade tumor budding, } \\
\text { larger nuclear diameter, higher mitotic count, } \\
\text { higher T, N and pathological stage }\end{array}$ & Worse RFS and OS \\
\hline $\begin{array}{l}\text { Pleomorphic } \\
\text { carcinoma }\end{array}$ & $40.0 \%$ & NA & $\begin{array}{l}\text { Trend of tumor necrosis (no statistical } \\
\text { difference) }\end{array}$ & Worse RFS and OS \\
\hline IMA & $56.1 \%$ to $72.3 \%$ & $\begin{array}{l}\text { Older age, absence of } \\
\text { GGO }\end{array}$ & NA & Worse RFS \\
\hline $\begin{array}{l}\text { Neuroendocrine } \\
\text { tumors } \\
\text { Typical carcinoid } \\
\text { Atypical carcinoid } \\
\text { SCLC } \\
\text { Large cell } \\
\text { neuroendocrine } \\
\text { carcinoma }\end{array}$ & $\begin{array}{l}16.0 \% \text { to } 20.5 \% \\
37.0 \% \text { to } 48.0 \% \\
46.0 \% \text { to } 83.3 \% \\
43.0 \%\end{array}$ & $\begin{array}{l}\text { NA } \\
\text { NA } \\
\text { NA } \\
\text { NA }\end{array}$ & $\begin{array}{l}\text { NA } \\
\text { Presence of necrosis, high mitotic count } \\
\text { NA } \\
\text { NA }\end{array}$ & $\begin{array}{l}\text { NA } \\
\text { Worse CIR } \\
\text { Worse CIR and LC-CID } \\
\text { Worse CIR and LC-CID }\end{array}$ \\
\hline $\begin{array}{l}\text { Lymphoepithelioma- } \\
\text { like carcinoma }\end{array}$ & $25.0 \%$ & NA & NA & No statistical difference \\
\hline
\end{tabular}

Abbreviations: ADC, adenocarcinoma; CIR, cumulative incidence of recurrence; GGO, ground-glass opacity; IMA, invasive mucinous adenocarcinoma; LC-CID, lung cancer-specific cumulative incidence of death; NA, not available; OS, overall survival; RFS, recurrence-free survival; SCLC, small cell lung carcinoma; SQCC, squamous cell carcinoma; STAS, spread through air spaces.

Positive STAS was observed to be associated with old age ( $>65$ years), male sex, smoking, abnormal serum carcinoembryonic antigen level, larger tumor size, nonlepidic (micropapillary/solid) predominant, cribriform component, moderate/poor differentiation, visceral pleural invasion, lymphovascular invasion, presence of nodal and distant metastasis and higher $\mathrm{T}, \mathrm{N}$ and pathological stage. ${ }^{16-21}$ In survival analyses, STAS was an independent risk factor for worse recurrence-free survival (RFS) and overall survival (OS). ${ }^{17,18,20}$ Similar results were identified in particular studies of stage I and stage III (N2) ADC. ${ }^{1,8,22-28}$ In SQCC, the incidence of STAS was generally lower than ADC, which was from $19.1 \%{ }^{4}$ to $40.3 \% .^{2}$ Positive STAS was observed to be associated with larger tumor size, lymphovascular invasion, tumor necrosis, high-grade tumor budding, larger nuclear diameter, higher mitotic count and higher $\mathrm{T}, \mathrm{N}$ and pathological stage. ${ }^{2-4}$ In survival analyses, STAS was a significant predictive factor for OS in univariate analysis, ${ }^{4}$ and an independent risk factor for recurrence and shorter RFS in multivariate analysis, especially for patients with stage I SQCC., ${ }^{2,4}$ In pleomorphic carcinoma, the incidence of STAS was reported to be $40.0 \%$. STAS tended to be more prevalent in tumors exhibiting necrosis, although this trend showed no statistical difference; meanwhile, survival analyses showed that patients with STAS experienced significantly worse RFS and OS, and multivariate analysis revealed that tumor STAS was an independent risk factor for both recurrence and shorter OS. ${ }^{5}$ Several meta-analyses have also been conducted based on the above-reported data, and the pooled results revealed that the presence of STAS suggested worse prognosis in non-small cell lung cancer collectively. ${ }^{11,29,30}$ 
In IMA, the incidence of STAS was from $56.1 \%$ to $72.3 \%$. IMA with STAS represented older age and absence of peripheral ground-glass opacity (GGO) on computed tomography (CT). In survival analyses, STAS was associated with reduced disease-free survival (DFS), but failed to be a significant prognostic factor; meanwhile, STAS was not associated with shorter OS. ${ }^{6,7}$

Neuroendocrine tumors also showed the phenomenon of STAS. In small cell lung carcinoma (SCLC), the incidence of STAS was reported to be $46.0 \%$ and $83.3 \%$. According to Toyokawa et $\mathrm{al}^{8}$ no significant associations between the presence of STAS and clinicopathologic characteristics were observed, and there seemed to be no significant difference in prognosis between patients with and without STAS. In another report, however, STAS was found to impact the cumulative incidence of recurrence (CIR) and to be an independent risk factor for lung cancerspecific cumulative incidence of death (LC-CID) in SCLC. ${ }^{9}$ In large cell neuroendocrine carcinoma, the incidence of STAS was reported to be $43.0 \%$, and STAS was also found to be an independent risk factor for CIR and LC-CID. In typical carcinoid, the incidence of STAS was from $16.0 \%$ to $20.5 \%$, while in atypical carcinoid, this incidence was from $37.0 \%$ to $48.0 \% .{ }^{9,31}$ STAS was reported to be associated with CIR in atypical carcinoid; meanwhile, prognostic analysis in typical carcinoid was not conducted due to the small number of events. ${ }^{9}$

In lymphoepithelioma-like carcinoma, the incidence of STAS was reported to be $25.0 \%$, and there seemed to be no significant difference in prognosis between patients with and without STAS. ${ }^{10}$

\section{Immunohistochemical and Molecular Features of Tumors with STAS}

The association between STAS and immunohistochemical or molecular characteristics has not been clearly explicated. In ADC, positive STAS was discovered to be significantly associated with tumor stroma metastasis-associated protein 1 expression level, ${ }^{17}$ but not significantly associated with programmed death ligand 1, SLX, thyroid transcription factor 1, napsin or CK7 expression as well as Ki-67 activity. ${ }^{19,24,32,33}$ In SQCC, the patients with STAS revealed an increased tendency for high vimentin and Ki-67 expression in comparison with the patients without STAS; however, expression of $\mathrm{p} 53$ and $\mathrm{E}$-cadherin was not associated with the status of STAS. ${ }^{2-4}$ Molecular characteristics were exclusively studied in ADC. STAS was frequently observed in tumors with ALK rearrangement, ROS1 rearrangement, BRAF mutations or wild-type HER2. ${ }^{16,19,20,34-36}$ Three articles reported the association between STAS and KRAS mutation; one study showed that STAS was frequently observed in tumors with KRAS mutation while the others showed no association. ${ }^{16,19,20}$ As for EGFR mutation, the results varied among different studies. One study showed that STAS was frequently observed in tumors with EGFR mutation, ${ }^{16}$ while another 3 studies demonstrated that STAS was associated with wild-type EGFR. ${ }^{19,20,35}$ Moreover, in another 3 studies, no correlation was observed between STAS and EGFR conditions. ${ }^{8,24,32}$

\section{Morphologic Subtypes of STAS}

The morphology of STAS contains several subtypes. In 2015, based on histologic features, Kadota and colleagues described 3 morphologic patterns of STAS: ${ }^{1}$ (1) micropapillary or ring-like clusters; (2) solid nests or tumor islands; (3) single cells. Among these subtypes, solid nests should be clearly identifiable within a low-magnification view, while the other two need to distinguish at a high-magnification view. ${ }^{5,37}$ STAS in ADC and pleomorphic carcinoma contained all the 3 subtypes, ${ }^{1,5,20,23,37}$ but STAS in SQCC contained exclusively solid nests pattern. ${ }^{2-4}$ In ADC and pleomorphic carcinoma, micropapillary or ring-like clusters pattern was always the most prevalent subtype, with an incidence from $58.2 \%$ to more than $80 \%,{ }^{23,37}$ while the other two subtypes were variable. Considering the subtypes of STAS and predominant histologic patterns of ADC (lepidic, acinar, papillary, micropapillary and solid), micropapillary clusters STAS was the most common type in all histologic patterns, single-cell STAS was a common pattern in lepidic predominant ADC, whereas solid nests STAS was a common pattern in solid predominant ADC. ${ }^{23}$ Meanwhile, other studies discovered that the micropapillary clusters and ring-like patterns were significantly associated with ALK rearrangements, and the solid nests pattern was associated with wild-type EGFR. ${ }^{20}$

\section{Significance of the Quantitative Evaluation of STAS}

Quantitative assessment of STAS has also been studied based on the number of cells or clusters of STAS. Patients were classified as having no STAS for cases without definite STAS, low STAS for cases with one to four single cells or clusters of STAS and high STAS for cases with five or more single cells or clusters of STAS. In the 2 studies of stage 
I ADC, ${ }^{24,38}$ STAS was assessed as low STAS in $18.3 \%$ and $17.4 \%$ cases, and high STAS in $29.3 \%$ and $38.0 \%$ cases, respectively. Higher STAS was found to be associated with solid predominant pattern, pleural invasion, lymphovascular invasion and larger tumor size. As for the prognosis, there was a significant association between increasing STAS and shorter RFS as well as OS, especially in the patients who underwent lobectomy. In SCLC, ${ }^{8} 20 \%$ and $63 \%$ cases were classified as having low and high STAS, while RFS and OS were not significantly different between negative/low STAS and high STAS patients.

Another method for quantitative evaluation is based on the distance between tumor surface and the farthest STAS from tumor edge. In $\mathrm{ADC}$, this distance, which was reported not to exceed the tumor diameter, ${ }^{39}$ was measured by a ruler with a range from 0.2 to $8.5 \mathrm{~mm}$ and a median from 1.0 to $1.5 \mathrm{~mm}$. When measured according to the number of alveolar spaces, this distance was from 1 to 58 with a median from 6 to $7^{1,23}$ Kadota and colleagues discovered that in $97 \%$ of the STAS-positive cases, tumor STAS was located beyond the first alveolar layer from the tumor edge. ${ }^{1}$ Meanwhile, when classified tumors as having limited STAS if tumor cells identified $<3$ alveoli away from the tumor edge whereas having extensive STAS if tumor cells were identified $>3$ alveoli away from the tumor edge, Warth and colleagues discovered that extensive STAS cases were more than limited STAS cases, and patients whose tumors showed limited STAS and patients whose tumors had extensive STAS had virtually similar DFS and OS. ${ }^{19}$ In SQCC, this distance was measured by a ruler with a range from 0.3 to $7.0 \mathrm{~mm}$ and a median from 1.0 to $1.4 \mathrm{~mm}$. When measured according to the number of alveolar spaces, this distance was from 1 to 40 with a median of $5 .^{2-4}$ Similar to ADC, no prognostic parameter was statistically different between the patients with limited and extensive STAS when a value of three alveolar spaces or $0.8 \mathrm{~mm}$ was used as a potential cutoff. ${ }^{2-4}$ Thus, although the distance between the tumor surface and the farthest STAS caused much attention, we thought this parameter did not influence the prognosis of patients.

\section{The Influence of STAS on Surgical Extent}

STAS also affected the prognosis according to a different surgical extent. The related studies were summarized in Table 2. STAS tends to have a more significant impact on the prognosis of stage I ADC patients who underwent sublobar or limited resection. ${ }^{1,24,26,40}$ Meanwhile, in stage I ADC with STAS, sublobar/limited resection was associated with a significantly higher risk of recurrence than lobectomy was, and similar results were observed in LC-CID. However, there was no significant difference in CIR between lobectomy and sublobar/limited resection in patients without STAS. ${ }^{27,36}$ In stage I SQCC, STAS was reported to be associated with worse RFS in both limited resection and lobectomy, while its impact on OS seems more obvious in limited resection. ${ }^{4}$ In all stage SQCC, STAS was also proved as a risk factor for worse prognosis, but its impact on different types of surgery is inconsistent in different studies. ${ }^{2,4}$ These results indicate that STAS should be paid more attention when presents in stage I ADC with limited resection. Whether these patients need extra lobectomy or postoperative treatment needs to be discussed. However, in SQCC, more data are needed to clarify the significance of STAS in different types of surgery.

Another issue in the sublobar or limited resection is the surgical margin distance, which is defined as the distance between the surgical staple margin and the nearest primary tumor edge. In stage I non-small cell lung carcinoma, no local recurrence occurred in tumors with a surgical margin distance greater than $2.0 \mathrm{~cm}$ regardless of the status of STAS. $^{37}$ Meanwhile, for patients with $\operatorname{ADC}(\leqq 3.0 \mathrm{~cm})$, a margin-to-tumor ratio (the ratio of surgical margin distance to tumor diameter) of 1 or higher was associated with a significantly lower risk of recurrence (particularly locoregional recurrence) than a margin-to-tumor ratio less than 1 in STAS-negative tumors; however, among patients with STAS-positive tumors, the risk of recurrence was high regardless of margin-to-tumor ratio. ${ }^{27}$ This finding indicates that the surgical margin distance or margin-totumor ratio might have a different impact on the prognosis of patients when the condition of STAS was different, and further studies are needed to discuss the safe distance when limited resection is performed on a STAS-positive patient.

\section{Radiology, Cytology and Frozen Section of STAS}

The diagnosis of STAS has also been studied in radiology, cytology and frozen sections. In CT manifestations of ADC, STAS-positive cases were significantly associated with a larger radiologic tumor diameter, the presence of vascular convergence, notch, pleural indentation, spiculation, central low attenuation, ill-defined opacity, air bronchogram, the absence of GGO and the high 
Table 2 Studies Concerning the Association Between Surgery Extent and STAS

\begin{tabular}{|c|c|c|c|}
\hline Reference & Tumor & Surgery Extent & Significance of STAS-Present compared with STAS-Absent \\
\hline \multirow[t]{2}{*}{ Kadota' } & \multirow[t]{2}{*}{ Stage I ADC } & Limited & $\begin{array}{l}\text { Higher risk of developing any types (locoregional or distant or both) of recurrence } \\
\text { Risk factor for recurrence in multivariate analysis }\end{array}$ \\
\hline & & Lobectomy & Not associated with an increased risk of recurrence \\
\hline \multirow[t]{2}{*}{ Toyokawa $^{24}$} & \multirow[t]{2}{*}{ Stage I ADC } & Limited & Shorter RFS and cancer-specific OS \\
\hline & & $\begin{array}{l}\text { Lobectomy or } \\
\text { bilobectomy }\end{array}$ & Shorter RFS but not cancer-specific OS \\
\hline \multirow[t]{2}{*}{$\operatorname{Ren}^{40}$} & \multirow[t]{2}{*}{ Stage IA ADC } & Limited & $\begin{array}{l}\text { Worse RFS and OS } \\
\text { Prognostic factor for RFS and OS in multivariate analysis }\end{array}$ \\
\hline & & Lobectomy & Worse RFS and OS \\
\hline \multirow[t]{4}{*}{ Kadota $^{2}$} & \multirow[t]{2}{*}{ All stage SQCC } & Limited & A tendency of worse RFS but not statistically significant \\
\hline & & Lobectomy or more & Lower 5-year RFS \\
\hline & \multirow[t]{2}{*}{ Stage I SQCC } & Limited & A tendency of worse RFS but not statistically significant \\
\hline & & Lobectomy & A tendency of worse RFS but not statistically significant \\
\hline \multirow[t]{4}{*}{ Yanagawa $^{4}$} & \multirow[t]{2}{*}{ All stage SQCC } & Limited & Worse RFS and OS \\
\hline & & Lobectomy & A trend of worse RFS and OS but not statistically significant \\
\hline & \multirow[t]{2}{*}{ Stage I SQCC } & Limited & Worse RFS and OS \\
\hline & & Lobectomy & Worse RFS and a not statistically significant trend of worse OS \\
\hline
\end{tabular}

Abbreviations: ADC, adenocarcinoma; OS, overall survival; RFS, recurrence-free survival; SQCC, squamous cell carcinoma; STAS, spread through air spaces.

percentage of solid component compared with STASnegative ones; meanwhile, the presence of notch, the absence of GGO and the high percentage of solid component were independent predictors of STAS..$^{35,41,42}$ In a single study of pulmonary ADC presenting as subsolid nodules, ${ }^{43}$ de Margerie-Mellon and colleagues found that compared with STAS-negative subsolid ADCs, the total average diameter, average and long-axis diameters of the solid component were larger, and the proportion of solid component diameter compared with total average diameter was higher in STAS-positive cases.

Cytologically, Isaka and colleagues ${ }^{44}$ found that tumor cell clusters detected in airway secretions of ADC showed similar morphologic features and a good correlation with STAS in routine histological sections. The sensitivity, specificity, and positive predictive value of airway secretion cytology for detecting STAS in routine tissue sections of ADC were $80 \%, 100 \%$, and $100 \%$, respectively.

As STAS might have an influence on the prognosis according to different surgical extension, the usage of frozen section in detecting STAS intraoperatively has been discussed. ${ }^{27,45}$ From the current results, the frozen section yielded sensitivity ranged from $59 \%$ to $86 \%$ and specificity ranged from $74 \%$ to $100 \%$. Based on these data, Walts et $\mathrm{al}^{45}$ considered that there were insufficient data to support intraoperative detection of STAS as a useful predictive feature to help stratify patients for lobectomy or sublobar resections. Due to the difficulties in selecting appropriate normal lung parenchyma sections for diagnosis based on gross observation and the interobserver difference in diagnosis of STAS on the frozen section, the reliability of using this method to predict the presence of STAS still remains to be proved.

\section{Disputes, Pathophysiology and Differential Diagnosis of STAS}

Although STAS has been widely studied and found a prognostic significance, the alternative view that this phenomenon is an artifact caused by mechanical forces during specimen handling is still in discussion. In recent years, Thunnissen and colleagues published several articles doubting STAS as an artifact but not a pattern of invasion based on the following reasons: ${ }^{46-48}$ (1) malignant loose tumor tissue fragments is a common phenomenon during the 
handling procedure of resection specimens; (2) most (93\%) loose tissue fragments were found in blocks sampled after cutting through tumor tissue and only a small minority was in the lung tissue sampled before the knife cut through the tumor; (3) the implication in STAS that tumor cells can live on air alone is biologically not plausible; (4) STAS-like artifact is simply seen in more discohesive tumors, that is more poorly differentiated with fewer intercellular adhesions, and this quality is responsible for the worse prognosis observed in these tumors. However, in the study published by Blaauwgeers et $\mathrm{al}^{47}$ the authors used the term "loose tissue fragments" and did not distinguish artifacts from STAS. Thus, these "loose tissue fragments" might contain both true STAS and true artifacts, and this might be an explanation for why most fragments were found in blocks sampled after cutting through tumor tissue. In 2017, Lu et al reported 3 cases demonstrating STAS was a real invasion pattern, ${ }^{49}$ in which STAS was observed without cutting through the main tumor. The mechanism for STAS survival has also been studied. In 2012, Onozato and colleagues found that isolated tumor islands detached in alveolar spaces were connected to other islands and/or the main tumor using three-dimensional (3D) reconstruction. ${ }^{15,50}$ This observation indicated that at least a small number of STAS clusters (especially those close to the main tumor) might not be truly spreading in the lung alveolar parenchyma. Recently, using 3D reconstruction and multiplex immunofluorescence staining, Yagi and colleagues revealed that STAS tumor cells could detach from the main tumor, migrate through air spaces, and reattach to the alveolar walls rather than appearing free floating; meanwhile, the reattached STAS cells were focally in close apposition to the pre-existing capillaries in the alveolar septa. ${ }^{51}$ Based on these results, Yagi et al suggested that dynamic blood vessel co-option, in which circumstance tumor cells exploited pre-existent vessels rather than inducing angiogenesis, ${ }^{52}$ could be a mechanism for STAS survival. And as some of the STAS cells might migrate beyond the limited resection margin in this way, patients with STAS who underwent limited resection demonstrated worse prognosis than those who underwent lobectomy.

As STAS can be easily confused with artifacts such as loose tumor tissue fragments, the differential diagnosis is fairly important. ${ }^{1,3,13,19,46-48,53}$ Artifacts that might be misdiagnosed as STAS and key points for differential diagnosis were concluded in Table 3. For these mimics, careful observation is needed and immunohistochemical staining may be useful in some issues.

Table 3 Artifacts That May Be Misdiagnosed as STAS

\begin{tabular}{|c|c|c|}
\hline Reference & Artifacts & Description and Key Points for Differential Diagnosis \\
\hline $\begin{array}{l}\text { Kadota } \\
\text { Warth }^{13} \\
\text { Thunnissen }^{46}\end{array}$ & $\begin{array}{l}\text { Tumor floaters/spread through } \\
\text { a knife surface }\end{array}$ & $\begin{array}{l}\text { - The most controversial issue for the presence of STAS. } \\
\text { - Presence of jagged edges of tumor cell clusters and linear strips of cells that are lifted off of } \\
\text { alveolar walls are indications for this artifact. } \\
\text { - Distribution of artificial loose tumor tissue fragments is haphazard, while the distribution of } \\
\text { STAS is consistent with the overall configuration of the circumferential tumor edge. }\end{array}$ \\
\hline Kadota' & Alveolar macrophages & $\begin{array}{l}\text { - The cytoplasm of macrophage is foamy containing pigment, and nucleus is small without } \\
\text { atypia. } \\
\text { - CD68 staining is helpful. }\end{array}$ \\
\hline Warth ${ }^{19}$ & Micropapillary pattern & $\begin{array}{l}\text { - STAS clusters separate from the main tumor. } \\
\text { - An imaginary line along the most peripheral edge of the main tumor may be helpful; cell } \\
\text { nests outside this line are considered as STAS. }\end{array}$ \\
\hline $\mathrm{Lu}^{3}$ & Tumor budding & $\begin{array}{l}\text { - Tumor budding is defined as the presence of isolated single cancer cells or a cluster of } \\
\text { cancer cells composed of fewer than five cells in the stroma at the outer edge of the tumor. } \\
\text { - Tumor budding is observed within the tumor stroma at the invasive front of the tumor, } \\
\text { whereas STAS is observed within air spaces. } \\
\text { beyond the edge of the tumor }\end{array}$ \\
\hline Pelosi $^{53}$ & $\begin{array}{l}\text { Spread of hyperplastic pulmonary } \\
\text { neuroendocrine cells }\end{array}$ & $\begin{array}{l}\text { - This artifact has been reported in ADC, typical carcinoid and atypical carcinoid. } \\
\text { - Free-floating aggregates of neuroendocrine cells are present in air spaces. } \\
\text { - Chromogranin A staining is helpful. }\end{array}$ \\
\hline
\end{tabular}

Abbreviations: ADC, adenocarcinoma; STAS, spread through air spaces. 


\section{Conclusion}

Above all, in this review, we comprehensively summarized current discoveries concerning STAS from multiple perspectives. STAS is a conspicuous spreading phenomenon of lung cancers indicating worse prognosis; nevertheless, the treatment strategy for patients with STAS remains to be discussed. Further studies are needed to elaborate whether a STAS-positive patient who underwent limited resection needs a second operation or postoperative adjuvant treatment. Meanwhile, although the nutrition resource has been studied, the internal mechanism of STAS formation is largely undiscovered. Whether the capability of detachment-migration-reattachment in STAS tumor cells is achieved at the time of primary tumorigenesis or in the progress of tumor development needs to be studied, and the related signal pathways or genetic alterations need to be explored. With this information, it may be possible to improve the prognosis of patients with STASpositive lung cancers.

\section{Abbreviations}

3D, three-dimensional; ADC, adenocarcinoma; CIR, cumulative incidence of recurrence; $\mathrm{CT}$, computed tomography; DFS, disease-free survival; GGO, ground-glass opacity; IMA, invasive mucinous adenocarcinoma; LCCID, lung cancer-specific cumulative incidence of death; OS, overall survival; RFS, recurrence-free survival; SCLC, small cell lung carcinoma; SQCC, squamous cell carcinoma; STAS, spread through air spaces.

\section{Data Sharing Statement}

The datasets used and/or analyzed in the current review are available from the corresponding author on reasonable request.

\section{Ethics and Consent Statement}

This study was approved by ethics committee of The Second Hospital of Jilin University (Changchun, China).

\section{Funding}

This work was supported by Science and Technology of Jilin Province, Jilin Province Key Laboratory (3D517K363429); The Role and Molecular Mechanism of EMT in the Resistance of ROS1-positive Lung Cancer (20180101014JC/ 3D518PS23429); Jilin Province Department of Finance Project (3D5197398429); Jilin Province Department of Finance Project (3D5197464429); and Youth Program of National Natural Science Foundation of China (3A4197642429). The research fund was used for data collection and paper publication.

\section{Disclosure}

The authors report no conflicts of interest in this work.

\section{References}

1. Kadota K, Nitadori J, Sima CS, et al. Tumor spread through air spaces is an important pattern of invasion and impacts the frequency and location of recurrences after limited resection for small Stage I lung adenocarcinomas. $J$ Thorac Oncol. 2015;10(5):806-814. doi:10.1097/JTO.0000000000000486

2. Kadota K, Kushida Y, Katsuki N, et al. Tumor spread through air spaces is an independent predictor of recurrence-free survival in patients with resected lung squamous cell carcinoma. Am J Surg Pathol. 2017;41(8):1077-1086. doi:10.1097/PAS.0000000000000872

3. Lu S, Tan KS, Kadota K, et al. Spread through Air Spaces (STAS) is an independent predictor of recurrence and lung cancer-specific death in squamous cell carcinoma. $J$ Thorac Oncol. 2017;12(2):223-234. doi:10.1016/j.jtho.2016.09.129

4. Yanagawa N, Shiono S, Endo M, Ogata SY. Tumor spread through air spaces is a useful predictor of recurrence and prognosis in stage I lung squamous cell carcinoma, but not in stage II and III. Lung Cancer. 2018;120:14-21. doi:10.1016/j.lungcan.2018.03.018

5. Yokoyama S, Murakami T, Tao H, et al. Tumor spread through air spaces identifies a distinct subgroup with poor prognosis in surgically resected lung pleomorphic carcinoma. Chest. 2018;154(4):838-847. doi:10.1016/j.chest.2018.06.007

6. Lee MA, Lee HY. P1.05-08 Spread Through Air Spaces (STAS) in invasive mucinous adenocarcinoma of the lung, incidence, prognostic impact, and predictive factors. $J$ Thorac Oncol. 2018;13(10):S543. doi:10.1016/j.jtho.2018.08.760

7. Ueda D, Tsutani Y, Ito M, Miyata Y, Okada M. P2.01-97 prognostic factors in resected lung mucinous adenocarcinoma, clinical and pathological features. $J$ Thorac Oncol. 2018;13(10):S702. doi:10.1016/j.jtho.2018.08.1151

8. Toyokawa G, Yamada Y, Tagawa T, et al. High frequency of spread through air spaces in resected small cell lung cancer. Anticancer Res. 2018;38(3):1821-1825. doi:10.21873/anticanres.12421

9. Aly RG, Rekhtman N, Li X, et al. Spread Through Air Spaces (STAS) is prognostic in atypical carcinoid, large cell neuroendocrine carcinoma, and small cell carcinoma of the lung. $J$ Thorac Oncol. 2019;14(9):1583-1593. doi:10.1016/j.jtho.2019.05.009

10. Yeh YC, Kao HL, Lee KL, Wu MH, Ho HL, Chou TY. Epstein-barr virus-associated pulmonary carcinoma, proposing an alternative term and expanding the histologic spectrum of lymphoepithelioma-like carcinoma of the lung. Am J Surg Pathol. 2019;43(2):211-219. doi:10.1097/PAS.0000000000001173

11. Chen D, Mao Y, Wen J, et al. Tumor spread through air spaces in non-small cell lung cancer, a systematic review and meta-analysis. Ann Thorac Surg. 2019;108(3):945-954. doi:10.1016/j.athoracsur. 2019.02.045

12. Ma K, Zhan C, Wang S, Shi Y, Jiang W, Wang Q. Spread Through Air Spaces (STAS), a new pathologic morphology in lung cancer. Clin Lung Cancer. 2019;20(2):e158-e162. doi:10.1016/j.cllc.2018.10.007

13. Warth A. Spread through air spaces (STAS), a comprehensive update. Transl Lung Cancer Res. 2017;6(5):501-507. doi:10.21037/ tlcr.2017.06.08

14. Giraud P, Antoine M, Larrouy A, et al. Evaluation of microscopic tumor extension in non-small-cell lung cancer for three-dimensional conformal radiotherapy planning. Int J Radiat Oncol Biol Phys. 2000;48(4):1015-1024. doi:10.1016/S0360-3016(00)00750-1 
15. Onozato ML, Kovach AE, Yeap BY, et al. Tumor islands in resected early-stage lung adenocarcinomas are associated with unique clinicopathologic and molecular characteristics and worse prognosis. Am J Surg Pathol. 2013;37(2):287-294. doi:10.1097/PAS.0b013e31826 $885 \mathrm{fb}$

16. Hu SY, Hsieh MS, Hsu HH, et al. Correlation of tumor spread through air spaces and clinicopathological characteristics in surgically resected lung adenocarcinomas. Lung Cancer. 2018;126:189-193. doi:10.1016/ j.lungcan.2018.11.003

17. Liu Y, Chen D, Qiu X, et al. Relationship between MTA1 and spread through air space and their joint influence on prognosis of patients with stage I-III lung adenocarcinoma. Lung Cancer. 2018;124:211-218. doi:10.1016/j.lungcan.2018.07.040

18. Qiu X, Chen D, Liu Y, et al. Relationship between stromal cells and tumor spread through air spaces in lung adenocarcinoma. Thorac Cancer. 2019;10(2):256-267. doi:10.1111/1759-7714.12945

19. Warth A, Muley T, Kossakowski CA, et al. Prognostic impact of intra-alveolar tumor spread in pulmonary adenocarcinoma. Am J Surg Pathol. 2015;39(6):793-801. doi:10.1097/PAS.0000000000000409

20. Lee JS, Kim EK, Kim M, Shim HS. Genetic and clinicopathologic characteristics of lung adenocarcinoma with tumor spread through air spaces. Lung Cancer. 2018;123:121-126. doi:10.1016/j.lungcan. 2018.07.020

21. Ding Q, Chen D, Wang X, et al. Characterization of lung adenocarcinoma with a cribriform component reveals its association with spread through air spaces and poor outcomes. Lung Cancer. 2019;134:238-244. doi:10.1016/j.lungcan.2019.06.027

22. Shiono S, Yanagawa N. Spread through air spaces is a predictive factor of recurrence and a prognostic factor in stage I lung adenocarcinoma. Interact Cardiovasc Thorac Surg. 2016;23 (4):567-572. doi:10.1093/icvts/ivw211

23. Dai C, Xie H, Su H, et al. Tumor spread through air spaces affects the recurrence and overall survival in patients with lung adenocarcinoma $>2$ to $3 \mathrm{~cm}$. J Thorac Oncol. 2017;12(7):1052-1060. doi:10.1016/j. jtho.2017.03.020

24. Toyokawa G, Yamada Y, Tagawa T, et al. Significance of spread through air spaces in resected pathological Stage I lung adenocarcinoma. Ann Thorac Surg. 2018;105(6):1655-1663. doi:10.1016/j.athoracsur.2018. 01.037

25. Yang L, Yang Y, Ma P, et al. Spread through air spaces predicts a worse survival in patients with stage I adenocarcinomas $>2 \mathrm{~cm}$ after radical lobectomy. $J$ Thorac Dis. 2018;10(9):5308-5317. doi:10.21037/ jtd.2018.09.22

26. Bains S, Eguchi T, Warth A, et al. Procedure-specific risk prediction for recurrence in patients undergoing lobectomy or sublobar resection for small $(</=2 \mathrm{~cm})$ lung adenocarcinoma, an international cohort analysis. J Thorac Oncol. 2019;14(1):72-86. doi:10.1016/j.jtho.2018.09.008

27. Eguchi T, Kameda K, Lu S, et al. Lobectomy Is associated with better outcomes than sublobar resection in Spread through Air Spaces (STAS)-positive T1 lung adenocarcinoma, a propensity score-matched analysis. $J$ Thorac Oncol. 2019;14(1):87-98. doi:10.1016/j. jtho.2018.09.005

28. Terada Y, Takahashi T, Morita S, et al. Spread through air spaces is an independent predictor of recurrence in stage III (N2) lung adenocarcinoma. Interact Cardiovasc Thorac Surg. 2019;29 (3):442-448. doi:10.1093/icvts/ivz116

29. Liu H, Yin Q, Yang G, Qie P. Prognostic impact of tumor spread through air spaces in non-small cell lung cancers, a meta-analysis including 3564 patients. Pathol Oncol Res. 2019;25(4):1303-1310. doi:10.1007/s12253-019-00616-1

30. Wang S, Hao J, Qian C, Wang H. Tumor spread through air spaces is a survival predictor in non-small-cell lung cancer. Clin Lung Cancer. 2019;20(5):e584-e591. doi:10.1016/j.cllc.2019.05.012

31. Altinay S, Metovic J, Massa F, et al. Spread through air spaces (STAS) is a predictor of poor outcome in atypical carcinoids of the lung. Virchows Arch. 2019;475(3):325-334. doi:10.1007/s00428-019-02596-8
32. Toyokawa G, Yamada Y, Tagawa T, et al. Significance of spread through air spaces in resected lung adenocarcinomas with lymph node metastasis. Clin Lung Cancer. 2018;19(5):395-400 e391. doi:10.1016/j.cllc.2018.04.002

33. Hara K, Mizuguchi S, Okada S, et al. Intensity of SLX predicts distance of tumor spread through alveolar spaces in stage I lung adenocarcinoma. Thorac Cancer. 2019;10(4):832-838. doi:10.1111/ 1759-7714.13008

34. Jin Y, Sun PL, Park SY, et al. Frequent aerogenous spread with decreased E-cadherin expression of ROS1-rearranged lung cancer predicts poor disease-free survival. Lung Cancer. 2015;89 (3):343-349. doi:10.1016/j.lungcan.2015.06.012

35. Kim SK, Kim TJ. Lung adenocarcinoma, CT features associated with spread through air spaces. Radiology. 2018;289(3):831-840. doi:10.1148/radiol.2018180431

36. Kadota K, Kushida Y, Kagawa S, et al. Limited resection is associated with a higher risk of locoregional recurrence than lobectomy in Stage I lung adenocarcinoma with tumor spread through air spaces. Am J Surg Pathol. 2019;43(8):1033-1041. doi:10.1097/PAS.0000000000001285

37. Masai K, Sakurai H, Sukeda A, et al. Prognostic impact of margin distance and tumor spread through air spaces in limited resection for primary lung cancer. J Thorac Oncol. 2017;12(12):1788-1797. doi:10.1016/j.jtho.2017.08.015

38. Uruga H, Fujii T, Fujimori S, Kohno T, Kishi K. Semiquantitative assessment of tumor Spread through Air Spaces (STAS) in early-stage lung adenocarcinomas. $J$ Thorac Oncol. 2017;12 (7):1046-1051. doi:10.1016/j.jtho.2017.03.019

39. Morimoto J, Nakajima T, Suzuki H, et al. Impact of free tumor clusters on prognosis after resection of pulmonary adenocarcinoma. J Thorac Cardiovasc Surg. 2016;152(1):64-72 e61. doi:10.1016/j. jtcvs.2016.03.088

40. Ren Y, Xie H, Dai C, et al. Prognostic impact of tumor spread through air spaces in sublobar resection for 1A lung adenocarcinoma patients. Ann Surg Oncol. 2019;26(6):1901-1908. doi:10.1245/ s10434-019-07296-w

41. Toyokawa G, Yamada Y, Tagawa T, et al. Computed tomography features of resected lung adenocarcinomas with spread through air spaces. J Thorac Cardiovasc Surg. 2018;156(4):1670-1676.e1674. doi:10.1016/j.jtcvs.2018.04.126

42. Koezuka S, Mikami T, Tochigi N, et al. Toward improving prognosis prediction in patients undergoing small lung adenocarcinoma resection, radiological and pathological assessment of diversity and intratumor heterogeneity. Lung Cancer. 2019;135:40-46. doi:10.1016/j. lungcan.2019.06.023

43. de Margerie-mellon C, Onken A, Heidinger BH, VanderLaan PA, Bankier AA. CT manifestations of tumor spread through airspaces in pulmonary adenocarcinomas presenting as subsolid nodules. J Thorac Imaging. 2018;33(6):402-408. doi:10.1097/RTI.0000000000000344

44. Isaka T, Yokose T, Miyagi Y, et al. Detection of tumor spread through airspaces by airway secretion cytology from resected lung cancer specimens. Pathol Int. 2017;67(10):487-494. doi:10.1111/pin.12570

45. Walts AE, Marchevsky AM. Current evidence does not warrant frozen section evaluation for the presence of tumor spread through alveolar spaces. Arch Pathol Lab Med. 2018;142(1):59-63. doi:10.5858/arpa.2016-0635-OA

46. Thunnissen E, Blaauwgeers HJ, de Cuba EM, Yick CY, Flieder DB. Ex vivo artifacts and histopathologic pitfalls in the lung. Arch Pathol Lab Med. 2016;140(3):212-220. doi:10.5858/arpa.2015-0292-OA

47. Blaauwgeers H, Flieder D, Warth A, et al. A prospective study of loose tissue fragments in non-small cell lung cancer resection specimens, an alternative view to "spread through air spaces". Am J Surg Pathol. 2017;41(9):1226-1230. doi:10.1097/PAS.0000000000000889

48. Blaauwgeers H, Russell PA, Jones KD, Radonic T, Thunnissen E. Pulmonary loose tumor tissue fragments and spread through air spaces (STAS), invasive pattern or artifact? A critical review. Lung Cancer. 2018;123:107-111. doi:10.1016/j.lungcan.2018.07.017 
49. Lu S, Rekhtman N, Eguchi T, Jones D, Adusumilli P, Travis W. P3.01-029 cases demonstrating Spread Through Air Spaces (STAS) reflects invasive growth and not an artifact, topic, morphology. J Thorac Oncol. 2017;12(1):S1137. doi:10.1016/j.jtho.2016.11.1595

50. Onozato ML, Klepeis VE, Yagi Y, Mino-Kenudson M. A role of three-dimensional (3D)-reconstruction in the classification of lung adenocarcinoma. Anal Cell Pathol (Amst). 2012;35(2):79-84. doi:10.1155/2012/684751

51. Yagi Y, Aly RG, Tabata K, et al. Three-dimensional histologic, immunohistochemical, and multiplex immunofluorescence analyses of dynamic vessel co-option of spread through air spaces in lung adenocarcinoma. $J$ Thorac Oncol. 2020;15(4):589-600. doi:10.1016/j. jtho.2019.12.112
52. Zhao C, Yang H, Shi H, et al. Distinct contributions of angiogenesis and vascular co-option during the initiation of primary microtumors and micrometastases. Carcinogenesis. 2011;32(8):1143-1150. doi:10. 1093/carcin/bgr076

53. Pelosi G, Nesa F, Taietti D, et al. Spread of hyperplastic pulmonary neuroendocrine cells into air spaces (S.H.I.P.M.E.N.T.S), A proof for artifact. Lung Cancer. 2019;137:43-47. doi:10.1016/j.lungcan.2019.09. 006

\section{Publish your work in this journal}

Cancer Management and Research is an international, peer-reviewed open access journal focusing on cancer research and the optimal use of preventative and integrated treatment interventions to achieve improved outcomes, enhanced survival and quality of life for the cancer patient.
The manuscript management system is completely online and includes a very quick and fair peer-review system, which is all easy to use. Visit http://www.dovepress.com/testimonials.php to read real quotes from published authors. 